\title{
Potter y Freire: diálogo de fundamentos teóricos para la educación bioética
}

Lourdes Quicutis Sánchez ${ }^{1}$

\section{Resumen}

El artículo presenta resultados de investigación encaminada a encauzar una educación bioética pertinente en el contexto universitario latinoamericano. Parte del presupuesto que es importante para el desarrollo de la educación bioética hacer consciente los puntos coincidentes entre la bioética de Van R. Potter y la pedagogía de Paulo Freire. Los objetivos propuestos son: la presentación profundizada del pensamiento de ambos autores; determinar los puntos teóricos coincidentes e identificar las bases teóricas necesarias para despliegues metodológicos que permitan superar las simplificaciones y la unidireccionalidad en la enseñanza bioética. El diseño metodológico acorde con una investigación teórica y una metodología cualitativa tiene como base la revisión bibliográfica, incluyendo técnicas como la entrevista a especialistas en la obra de los autores. Las conclusiones muestran que se logró establecer diálogo entre ambas teorías, cuya lógica dialéctica común se constituye en un pensamiento crítico sobre la forma en que tradicionalmente se manejó el conocimiento.

Palabras-clave: Educación. Bioética. Toma de conciencia. Diálogo. Conocimientos.

\section{Resumo}

\section{Potter e Freire: diálogo dos fundamentos teóricos para a educação bioética}

$\mathrm{O}$ artigo apresenta resultados de pesquisa realizada para subsidiar uma educação em bioética pertinente ao contexto universitário latino-americano. Parte do pressuposto que é importante para o desenvolvimento da educação bioética tornar conscientes os pontos coincidentes entre a bioética de Van R. Potter e a pedagogia de Paulo Freire. Os objetivos propostos são: apresentação aprofundada do pensamento de ambos os autores; determinação dos pontos teóricos coincidentes e identificação das bases teóricas necessárias para modelos metodológicos que permitam superar as simplificações e a unidirecionalidade do ensino bioética. 0 desenho metodológico concilia uma pesquisa teórica e uma metodologia qualitativa, tendo como base a revisão bibliográfica, incluindo técnicas como entrevista com especialistas na obra destes autores. As conclusões mostram que se conseguiu estabelecer diálogo entre ambas as teorias, cuja lógica dialética comum se constitui em pensamento crítico sobre a forma que tradicionalmente se aborda o conhecimento.

Palavras-chave: Educação. Bioética. Conscientização. Diálogo. Conhecimento

\section{Abstract \\ Potter and Freire: dialogue of theorical fundamental for bioethics education}

The paper presents results of research conducted to subsidize education in bioethics relevant to the Latin American university context. It assumes that it is important for the development of bioethics education make aware the coincident points between Van R. Potter bioethics and the pedagogy of Paulo Freire. The proposed objectives are: a thorough presentation of the thought of both authors; determine coincident theoretical points and; identify the theoretical foundations necessary for methodological models that allow overcoming the simplifications and unidirectionality of bioethics teaching. Our methodology combines a theoretical research and a qualitative methodology, based on the literature review, including techniques such as interviews with experts in the work of these authors. The findings show that dialogue could be established between the two theories, whose common dialectical logic constitutes critical thinking about the way that knowledge is traditionally approachedThis article presents the results of an investigation, to put an education on the right track pertinent bioethics in the university Latin American context.

Key words: Education. Bioethics. Awareness. Dialogue. Knowledge.

1. Maestra Iqsanchez@giron.sld.cu - Departamento de Filosofía, Facultad de Ciencias Médicas Victoria De Girón, La Habana, Cuba.

Correspondência

Avenida $237 \# 13603$ entre 136 y 138, Bauta/Artemisa, Cuba.

Declara não haver conflito de interesse. 
La Educación Universitaria actual aún se desarrolla bajo el predominio del paradigma clásico, unidireccional y tradicional en la construcción de conocimientos. Este paradigma de enseñanza considera que el estudiante en el proceso de enseñanza-aprendizaje no tiene por qué ir más allá de la recepción pasiva del contenido. La trasgresión de esta línea se limita a la expresión de dudas sobre el contenido en estudio, para entender mejor lo que el profesor está explicando, o para dar respuesta a preguntas formuladas por el docente con la intención de profundizar en elementos relacionados con un tema determinado.

El mismo paradigma clásico determina que el tema en cuestión en la enseñaza ha sido establecido en un programa diseñado centralmente, y que en pocos casos es reajustado o adaptado por el profesor a las circunstancias concretas en que se está impartiendo el contenido. Así, estos son básicamente los elementos que caracterizan una educación tradicional y unidireccional enmarcada por la verticalidad en el repaso del conocimiento y la centralidad en la definición de lo que sea esto conocimiento. Como paradigma pedagógico esta forma de ejercicio de la pedagogía está llamada a desaparecer en un mundo que necesita cada vez más del respeto a la diversidad de criterios, culturas y conocimientos.

Esta "fórmula" básica es aplicada a todas las disciplinas y es también un hecho que la Educación Bioética escolarizada no escapa a este paradigma. Pero se debe considerar en este caso el agravante que si la educación universitaria en general se pueda dar a este lujo, a la Bioética no le está permitido dárselo pues entra en total contraposición con el objetivo, la intención y el propio contenido de los conocimientos que la disciplina divulga y defiende. O sea, no estimula la reflexión indispensable a la construcción del conocimiento.

Para reafirmar lo anteriormente dicho es necesario declarar que desde la década de los $90 \mathrm{del}$ siglo pasado la bioética latinoamericana ha comenzado un viraje hacia el reconocimiento de la centralidad y el formar parte de la racionalidad de nuevo tipo, de la concepción global, holística y profunda de Van Rensselaer Potter, ${ }^{1,2}$, para profundizar en el desarrollo de la bioética global sustentable en América Latina ${ }^{3}$. En este esfuerzo intelectual están involucrados diversos pensadores del continente, los cuales desarrollaran perspectivas autóctonas, como José R. Acosta en su libro Los árboles y el bosque ${ }^{4}$, el cual procura adaptar su texto esta nueva suerte de reflexión Bioética, considerando específicamente el contexto bioético cubano.
El estudio aquí presentado es producto de investigaciones académicas para la obtención del grado de maestría a cual explota las interfaces entre los dos pensadores. Busca también, adicionalmente, estimular estos nuevos caminos de la reflexión bioética a partir del aporte pedagógico de Paulo Freire ${ }^{5-10}$.

\section{Contexto intelectual y método}

La enseñanza de la bioética no puede estar de espaldas a este resurgir del pensamiento potteriano que es incompatible con ese paradigma $y$ demanda cambios fundamentales en el modo de enseñar-aprender. En este sentido salta a la vista en el pensamiento pedagógico latinoamericano la obra de Paulo Freire que proyecta en esencia una pedagogía donde la característica esencial del proceso de enseñanza-aprendizaje es el dialogo abierto y claro entre el estudiante y el profesor. Este diálogo deberá estar marcado por un respeto mutuo entre educadores y educandos, que llega a aceptar la flexibilidad de los contenidos a partir de las necesidades y conocimientos anteriores del estudiante.

El objetivo medular aquí es el proceso de concientización del educando, que le permitirá instruirse e ir desnaturalizando lo que parece natural y no lo es ${ }^{11}$ al decir de Esther Pérez. La concientización tendrá lugar en su comportamiento profesional y social en general, como toma de conciencia de su mundo interior y exterior, como un actuar en consecuencia en busca de su desarrollo. Así mismo, investigaciones realizadas han mostrado que las obras de Freire y Potter comparten ideales de conocimiento:

La reforma educativa profunda es parte de la revolución contemporánea del saber, y ha de beber en aquellas fuentes que aportan al nuevo saber humano. Es precisamente esa coincidencia fundamental entre Morin, Potter y Freire como portadores de un nuevo saber, la que hace sus ideas cercanas e ineludibles al plantearnos una reforma profunda de la enseñanza $^{12}$.

Sin embargo como punto de partida de la presente investigación se realizaron entrevistas a especialistas de ambos pensamientos y se evidenció que entre los investigadores que trabajan la perspectiva teórica de Potter no siempre existe un conocimiento profundo de la pedagogía freireana y viceversa. Así, autores fundamentales de la bioética global, reconocen el valor de la obra de Freire, pero no han explorado las posibles coincidencias y divergencias teóricas entre Potter y Freire y por ende no han vin- 
culado la enseñanza bioética a la pedagogía freireana. Por otra parte, los investigadores de la obra de Freire, reconocen no tener prácticamente ningún conocimiento de la obra de Potter; por lo que es evidente que no han concientizado las coincidencias de ideales y las potencialidades de la pedagogía freireana en la educación bioética.

Más allá de la constatación de la separación y falta de diálogo entre los estudiosos de la obra de Potter y Freire, la educación bioética demanda un ejercicio pedagógico específico, donde el diálogo de saberes es una necesidad imperiosa. Queda claro que la pedagogía tradicional deja poco espacio a este tipo de diálogo y se requiere indagar en las posibilidades que ofrecen otras alternativas pedagógicas. A partir de todo lo señalado hasta aquí se considera que existe en la teoría y la práctica pedagógica actual el fundamento básico para desplegar una investigación encaminada a explorar los vínculos entre el pensamiento de Potter y el de Freire.

La afirmación anterior se concreta en el hecho de que en la actualidad el desarrollo de la bioética demanda una mayor atención a los problemas relacionados con la educación bioética. Para encauzar una educación bioética pertinente en el contexto universitario latinoamericano, es importante hacer conscientes las coincidencias y diferencias entre la bioética de Potter y la pedagogía freireana, lo que tendría a su vez una repercusión importante en la educación universitaria en general. A partir de lo cual se plantearon los siguientes objetivos:

- Identificar los elementos de la bioética potteriana como una disciplina de nuevo tipo;

- Identificar los elementos teóricos fundamentales de la pedagogía freireana;

- Determinar los elementos teóricos existentes en la pedagogía freireana y la obra de Potter que permitan desarrollar un diálogo enriquecedor entre ambas teorías;

- Identificar las bases teóricas necesarias para despliegues metodológicos que permitan superar las simplificaciones y unidireccionalidad dominadora de la enseñanza universitaria y bioética en general.

Para lograr el cumplimiento de los objetivos anteriores se desarrolló un diseño metodológico basado en una investigación teórica que se apoya en la revisión bibliográfica, desde las bondades auxiliares del método hermenéutico para la comprensión de significados de términos y conceptos. Se emplearon procedimientos inductivos para elaborar generalizaciones a partir de la exploración empírica. Se proce- dió además a un análisis contextualizado de las propuestas teóricas de Potter y Freire; y a la identificación de semejanzas y diferencias en el plano teórico. Así la investigación que generó este artículo puede ser caracterizada como cualitativa, con encuesta basada en entrevista sobre la obra de los autores en asociación a la recolección de datos bibliográficos de esta misma obra.

Se utilizó así mismo el discurso teórico de la bioética potteriana y de la pedagogía freireana en calidad de metodología que sentará las bases teóricas necesarias para una educación bioética pertinente en el contexto universitario latinoamericano. Este cruce teórico-metodológico, de conjunto con el método hermenéutico permitirá esclarecer muchos elementos de coincidencia ocultos tras la terminología diferente.

La realización de esta investigación impuso la consulta exhaustiva de la obra de ambos autores, que si bien está fundamentalmente centrada en la concepción de bioética global (Potter) y de la pedagogía de la liberación (Freire), parte por reconocer la unidad orgánica que constituyen las respectivas propuestas. Esa unidad radica en los ideales que sirven de base a la construcción de ambas teorías. Como los ideales no son evidentes y se encuentran ocultos, puede ocurrir que quienes conocen una teoría, no hayan meditado acerca de los ideales que le sirven de base y esto explicaría la aparente distancia que existe entre lo que Potter y Freire nos proponen desde contextos y planteamientos teóricos que efectivamente difieren en su forma específica.

Entre las técnicas aplicadas se encuentra la entrevista semi-estructuradas a especialistas en cada uno de los pensamientos, siguiendo como criterio de selección el que su experiencia profesional y o estudios realizados demuestren que poseen un alto conocimiento sobre la obra del autor en cuestión. Las entrevistas fueran hechas con 8 especialistas en bioética (bajo criterio de saturación de las respuestas obtenidas) y con 9 especialistas en Freire (total de especialistas localizados), configurando un total de 17 especialistas. Fueran aplicada a especialistas radicados en La Habana, Cuba, durante el periodo comprendido entre los meses de enero y junio del 2011. Solo con dos de los especialistas en Freire se utilizó la vía del correo electrónico y las 15 restantes se realizaron de forma presencial con grabaciones que permitieran ser retomadas una y otra vez.

Las entrevistas se validaron a su vez por un panel de expertos en ambos pensamientos, con el objetivo de buscar los elementos existentes en cada una de estas teorías que puedan servir de análisis 
común entre ambas y no hayan sido detectadas o poco fundamentadas por la autora. Además se aplicaron técnicas puramente teóricas como:

- Análisis de contenido;

- Análisis comparativo;

- La teoría convertida en herramienta metodológica. Se utilizará la teoría de cada autor estudiado como herramienta para adentrarnos en la obra del otro autor.

Como antecedentes de este trabajo se encuentran los estudios que han vinculado el pensamiento bioético y el pensamiento complejo, que iniciaron el diálogo de la bioética con otros saberes que forman parte de la revolución contemporánea del saber y los estudios realizados por Díaz acerca de los vínculos entre Morin, Potter y Freire, en especial el artículo: Dialogo de saberes para una reforma del pensamiento y la enseñanza en América Latina: Morin-Potter-Freire ${ }^{13}$.

\section{Potter y Freire: coincidencias de fundamentos teóricos}

Van Rensselaer Potter y Paulo Freire nacido uno en el 1911 y el otro en 1921, respectivamente, son dos pensadores del siglo $\mathrm{XX}$ que desarrollaron al unísono sus teorías, pues ambos desplegaron el mayor peso de su obra durante la segunda mitad del siglo. Más allá de la similitud de sus fechas de nacimiento, pasaremos a precisar los elementos de diálogo existentes en los fundamentos teóricos que sustentan la bioética de Potter y la pedagogía de Freire.

Para lograr una comprensión real del tema debemos partir del resultado que nos brindó la aplicación del método hermenéutico, al permitirnos el abordaje de conceptos propuestos desde una de las teorías pero susceptibles de ser utilizados en ambas y que de hecho utilizaremos indistintamente, para la mejor visión de los elementos teóricos comunes existentes en las ideas que defienden los dos pensamientos. Consideremos los siguientes:

\section{Desarrollo humano}

Este concepto se utiliza en Freire desde una perspectiva más cerrada, al contemplarlo como la evolución económica, política y social inmediata, de una sociedad concreta. En Potter se analiza el desarrollo humano desde una perspectiva de largo alcance al vislumbrar esa evolución como el avance en la lucha por la supervivencia humana. Sin embargo el que existan diferencias en las dimensiones en que se analiza el desarrollo humano no incide en ningún momento en una contraposición entre el concepto de uno y de otro. Son complementarios, porque se hace imposible el logro de esa sabiduría de largo alcance que pretende Potter sin un sustento en acciones económicas, políticas y sociales a corto plazo.

A partir de lo antes expuesto es posible realizar una complementación de ambas concepciones concluyendo que al hablar de desarrollo humano, tanto en Freire como en Potter, nos referimos a la evolución económica, política y social personal, nacional, regional y planetaria que permita garantizar la supervivencia humana.

\section{Supervivencia humana}

Este concepto propuesto también desde la concepción de Potter, se expresa en Freire con la perspectiva del oprimido que tiene ante sí la urgente tarea de desarrollar el conocimiento y los valores para alcanzar la libertad que le puede devolver la condición de humano. Es visto en Potter, esencialmente, desde la necesidad que tiene la especie de evitar su desaparición producto del mal manejo de los conocimientos. Pudiera parecer que la connotación en ambas teorías es diferente, si no se entiende la concepción sobre la mera supervivencia y la supervivencia miserable de Potter y su reconocimiento del hecho de que estas supervivencias forman parte de la realidad de países y pueblos contemporáneos.

Reflexiona Potter además, en el hecho de que en estas regiones, procesos sociales como la conquista y colonización han constituido la causa principal del tránsito dramático de pasar de una mera supervivencia hacia una supervivencia miserable.

\section{Fundamentos teóricos comunes}

Como punto de partida entendemos necesario declarar el hecho de que ambas teorías tienen como cuestionamiento básico ¿cómo manejar el conocimiento? A partir de este punto inicial existe como fundamento en una y la otra una interrogante epistemológica que sirve de sustento a todas las coincidencias posteriores que podamos analizar.

En relación con lo anterior se puede plantear que estos dos autores expresan un pensamiento crítico sobre la forma en que tradicionalmente se concibió y aún se concibe la producción del conocimiento, considerando si es la correcta para producir o manejarlo. Al decir de Carlos Delgado ambos tienen una apertura crítica al resto de los saberes y las prácticas humanas ${ }^{14}$. Este pensamiento crítico 
se evidencia al plantearse como base de sus teorías la lucha contra ideales de conocimientos no compatibles con las exigencias de transformación del mundo de finales del siglo XX y principios del XXI.

Nos encontramos entonces en el caso de Freire ante un luchador contra lo que él llama "pedagogía bancaria" en contraposición con el nuevo ideal de pedagogía que se propone liberadora y en el caso de Potter se contrapone la producción de conocimientos disciplinarios contra un conocimiento transdisciplinario y complejo. Se establece así en la educación bancaria de Freire una clásica relación de poder y dominación que Potter en su bioética global analiza ha prevalecido en el control del conocimiento por las ciencias dentro de la sociedad y que ha llevado al comprometimiento del futuro. Queda claro entonces que en ambos hay un evidente antagonismo con las formas clásicas de cómo manejar el conocimiento a partir de propuestas no clásicas.

Desde esta contraposición con el ideal clásico de cómo manejar el conocimiento surge, como un hecho demostrado, que ambos pensadores presentan como base de su crítica la necesidad del reconocimiento a la diferencia de conocimientos, culturas, credos, puntos de vistas, concepciones e ideas sobre la realidad. Aceptación a la diferencia que funge como punto de partida para que cada un de estos pensamientos se condicionen con un criterio de inclusión que no se permite bajo ningún concepto la idea de exonerar conocimiento o valoración alguna, muy por el contrario sumar cada vez más en la búsqueda de la sabiduría de cómo manejar el conocimiento. En resumen podemos valorarlos como sistemas integradores e inclusivos.

Es a su vez este carácter integrador punto de partida para hacer valer el que a pesar de ello no exista superioridad ni dominación posible de unos sobre otros por lo tanto es necesario el respeto entre todos los saberes como una condición humana más, aspecto este que hace valer el carácter democrático de las teorías analizadas. Esto carácter democrático se expresa a partir de que ninguno de los dos ignora la existencia - cada vez con más fuerza - de nuevos actores sociales con quienes es imprescindible contar mucho más si de problemática educativa se trata. El carácter democrático se expresa de inicio a fin en la obra de los dos autores, con independencia de la evolución de sus pensamientos, porque para ambos es la contribución de todos y cada uno desde sus potencialidades y posibilidades lo único que permitirá el desarrollo humano.

Así mismo las coincidencias en sus pensamientos se extienden al análisis que ambos autores hacen de las inercias que impiden el desarrollo humano detectadas tanto por Freire como por Potter. Entre estas inercias se encuentra la no existencia de diálogo entre los diferentes conocimientos. Tanto uno como el otro autor entienden que para poder lograr una real sabiduría es necesario que todos los conocimientos alcanzados por la humanidad, desde las diferentes aristas en que este ha tenido la posibilidad de desarrollarse, conversen. Logrando en este diálogo un encuentro con la sabiduría humana, para a partir de ahí fomentar el desarrollo humano de frente hacía el futuro. El diálogo se presenta entonces tanto en Freire como en Potter como la piedra angular para el logro de la supervivencia humana.

Si de análisis de coincidencias entre ambos pensamientos se trata será mucho más apremiante que con ningún otro elemento el recurrir constantemente al diálogo para lograr entender y explicar el resto de sus coincidencias. El diálogo a su vez se hace extensivo en la bioética de Potter con la ética del discurso y en la pedagogía de Freire con la educación problematizadora. En ambos casos lo más significativo es el contenido reflexivo de este, a punto de ser considerado no como el intercambio pasivo de ideas y conocimientos sino como un intercambio prolifero y fecundo para ambas partes, en la búsqueda de la verdad. Así mismo esta concepción de diálogo reflexivo lleva implícito la posibilidad de que nuestros conocimientos estén errados y puedan ser transformados por los conocimientos ajenos.

Surgiendo la incertidumbre como otro de los elementos que enlazan a un pensamiento con otro, propuesto incluso desde la teoría de la complejidad, pero que tanto Freire como Potter aceptan. Ambos pensamientos abogan por el reconocimiento del conocimiento ajeno. Defendiendo además la idea de que los controladores clásicos del conocimiento (científicos y educadores) admitan no poseer de forma absoluta la verdad en sus manos. Por lo tanto científicos y educadores deben estar impuestos de que sus conocimientos pudieran estar errados y se pudieran corregir desde otra perspectiva.

A partir de lo cual la incertidumbre se concreta en Potter en su análisis acerca de los dos cuestionamientos básicos de su pensamiento, expuestos en el capítulo anterior, ¿qué tipo de futuro tenemos por delante? y ¿tenemos alguna opción? Relacionado con estas interrogantes vemos también en Potter la necesidad de asumir la respuesta a las mismas con humildad, que significa permitirnos encontrar respuestas en la búsqueda de la sabiduría de largo alcance incluso entre la sabiduría existente en otras especies. Por otra parte en Freire se manifiesta la 
incertidumbre al pretender que la educación liberadora sea un cuestionamiento constante de nuestro yo en primera instancia para después permitirnos cuestionarnos el mundo exterior.

Ambas teorías en esencia desarrollan la incertidumbre en el conocimiento sobre el sustento de que nuestros conocimientos se encuentran inmersos en una dialéctica que demanda un enriquecimiento y perfeccionamiento persistente. Dicha incertidumbre en el conocimiento es solo posible si se actúa con humildad. La humildad es condición indispensable en la creación y aplicación del conocimiento tanto para Potter como para Freire. Ambos exigen que se respete el conocimiento ajeno a partir de aceptar que el conocimiento propio no tiene que ser el único, el más completo o exacto lo que implica una gran cuota de humildad.

La humildad cognoscitiva es un concepto coincidente. Sí Freire la analiza como la necesidad de que el educador entienda su posibilidad de aprender durante el acto educativo, el pensamiento potteriano la reclama con respecto a la labor del científico, que se cree poseedor de verdades, y carente de humildad habilita la pregunta “¿podría estar equivocado?”, convocando ambos de esta manera a la prudencia en las acciones humanas. Aunque la utilización del término de humildad en los dos pensamientos es fácil de entender, sobre todo si se interrelaciona con elementos abordados con anterioridad como la aceptación de las diferencias, posibilidades de diálogo entre diferentes e incertidumbre, es necesario tener mucho cuidado cuando se lo utiliza porque la riqueza de nuestro idioma nos pone trampas muy a menudo. Con facilidad se cambia el significado de las palabras en relación con creencias, ideales e idiosincrasia y la humildad, palabra proveniente de lo humilde, se relaciona no en pocas ocasiones con la pobreza. En este caso para nada ninguno de los autores consideran o permiten considerar se valore algún conocimiento como pobre.

Desde sus teorías cada uno defiende el que toda persona tenga el nivel cultural que tenga, la profesión, especialización, creencia, grupo social o concepción filosófica sea escuchada en la reflexión y se tome de sus conocimientos lo que pueda aportar a la búsqueda de la verdad. Los dos autores abogan por no menospreciar ningún conocimiento, porque el menosprecio a los argumentos que brinda cualquier parte de la realidad sería ignorar la humildad que propicia el avance del conocimiento. Esta idea de autoridad de un conocimiento sobre otro se expresa en el ideal clásico al aceptar al conocimiento de las ciencias concretas como el único exacto y verdadero.
Nótese que una vez más, la coincidencia entre Potter y Freire no es casual ni resultado de compartir fuentes de información. Resulta de compartir ideales, en este caso, el enlace entre autonomía, humildad y responsabilidad es simultáneamente un ejercicio de toma de conciencia de los límites y las posibilidades del conocimiento con que desplegamos y los valores con que justificamos nuestras acciones.

\section{El carácter dialógico de las teorías}

Para seguir avanzando en los puntos que hacen coincidentes las teorías de Freire y Potter tenemos que volver a referirnos, como apuntábamos al inicio, al eje central de ambas teorías. Definido este eje central como su carácter dialógico partiremos de él para explicar que en la exigencia de que ocurra ese diálogo, (que Potter define con el adjetivo de puente), se entrelaza otra idea común: Ambos reclaman la necesidad y urgencia de la unión o interrelación entre las ciencias, la tecnología, las humanidades en general y la ética en particular. Esto ocurre de forma tal que en la búsqueda del conocimiento intervengan criterios de las ciencias concretas y el resultado de sus estudios "objetivos" de la realidad y los criterios y valoraciones "subjetivas" de las humanidades.

Visto este elemento en Potter desde su propuesta de que la bioética se constituya en una disciplina de nuevo tipo transdisciplinar que rompa con la dicotomía entre ciencias y humanidades. En Freire desde la exigencia de que en las escuelas no solo se enseñen conocimientos relacionados con la preparación científica y técnica del estudiante sino que además se le prepare para realizar valoraciones humanas relacionadas con aspectos de toda la realidad en que el educando se desenvuelve. Es fácil entonces percatarse que el logro de una educación que forme especialistas en una nueva disciplina transdisciplinar solo se podrá lograr en la escuela freireana que enseña conocimientos científicos a la par que forma valores humanos. Una escuela capaz de analizar la ciencia y la técnica a la luz de la responsabilidad y la humildad cognoscitiva, en bien del futuro humano.

Las propuesta de transformaciones sociales de ambos; desde la propuesta de una nueva educación comprometida con la liberación de los oprimidos, en Freire; a la creación de un nuevo ideal científico que busque una sabiduría de largo alcance, de Potter; se basan en un análisis de la relación existente entre el elemento económico, el político y el social en cualquier esfera o fenómeno de la sociedad. A partir de aquí Freire desarrolla un método de alfabetización 
que no se conforma con enseñar a leer y a escribir, sino que busca producir un cambio efectivo y real en el hombre y en su autocomprensión personal y en la comprensión del mundo que le rodea.

Se plantea entonces la pedagogía de Freire como un método participativo que se autodesarrolla en la medida en que el hombre se compromete con él. Pedagogía que tiene su máximo esplendor en el proceso de concientización, proceso propuesto como el objetivo máximo de su teoría.

\section{Bases para el cambio en la educación bioética}

Estamos ahora en condiciones de establecer cuáles serían las bases teóricas que permitirán la elaboración de futuros despliegues metodológicos que propicien el enrumbar la educación bioética como un proyecto educativo que se desarrolle en correspondencia con los postulados básicos de la propia disciplina y sobre las bases de la concepción educativa de educador-educando y educandoeducador, desarrollada por Freire. Al determinar los supuestos metodológicos necesarios para una educación en bioética no clásica, sobre la base de los presupuestos freireanos se entiende que los mismos son susceptibles de concentrarse en varias premisas que contendrán los supuestos metodológicos que proponemos considerar: 1) Premisas epistemológicas; 2) Premisas pedagógicas; 3) Premisas éticas.

\section{Análisis de las premisas}

\section{Premisas epistemológicas}

La educación en bioética debe asumir un ideal epistemológico en concordancia con el ideal no clásico: asumir la perspectiva del educando. Esto presupuesto que implica en adaptar el conocimiento a las circunstancias concretas en que existe, se desarrolla, actúa y desempeñara la labor cognoscitiva el educando; a partir de que nadie asimila y produce conocimiento desde una perspectiva aislada de sus circunstancias biopsicosociales. Se debe contextualizar el proceso de obtención de conocimiento.

\section{- Eliminar el ideal de fragmentación y simplifica- ción}

Este postulado significa que es necesario acabar con la idea de una educación enclaustrada en el ámbito de una institución y los muros de una construcción. La educación debe ubicarse como un proceso que se desarrolla en todos los espacios de socialización existentes sin distinción o jerarquía entre los mismos.

\section{- Aceptar la incertidumbre del conocimiento}

Educar en bioética significa ante todo que se está impuesto de la posibilidad de que nuestros conocimientos estén errados o sean incompletos, que existen otras perspectivas desde las que se puede analizar un mismo fenómeno a partir de la cual se puede llegar también a la verdad.

\section{- La unidad interna entre conocimiento y valor}

El proceso educativo no debe entenderse como un proceso en el que se van a recepcionar conocimientos científicos. $O$ un proceso en el que se aprenderá a analizar y actuar en la sociedad. Sino que debe percibirse como un acto de total comprensión de la realidad compuesto por elementos científicos y elementos valorativos. Elementos que interrelacionados nos permitirán adquirir la sabiduría necesaria para manejar ese conocimiento con humildad y responsabilidad. Nos permitirán actuar de forma pertinente.

\section{- Reconocer las posibilidades epistémicas}

Es imprescindible que la educación en bioética pueda reconocer las posibilidades epistémicas de todos los seres humanos, que pueden pensar el conocimiento e intentar conocer el conocimiento. La Educación en bioética debe encontrarse impuesta del ideal que acepta como condición humana la posibilidad de brindar conocimiento y recibirlo a partir de que la experiencia, la interrelación diaria con la realidad, le brinda siempre al individuo un conocimiento que le permite aportar al proceso.

\section{Premisas pedagógicas}

\section{- Reconocimiento a la diversidad}

Cualquier propuesta metodológica para la educación bioética debe tener en cuenta que todos los educandos no tienen necesariamente los mismos intereses, aspiraciones y necesidades. La educación bioética debe estar imbuida de la posibilidad y la necesidad de que el educador realice una investigación previa. Investigación que le permitirá guiar el proceso de creación de conocimientos a partir de palabras o temas generadores, como recomendaba Freire, o a partir de una identificación de elementos contextuales que sirvan al mismo propósito. 


\section{- Carácter dialógico}

Las palabras o temas generadores deben ser el punto de partida o fuerza movilizadora del diálogo como método fundamental o básico que se utilizará en la búsqueda de la verdad, como respuesta a las problemáticas que presentará el educador.

\section{- Carácter problematizador}

El educador no debe presentar nunca el conocimiento que posee como acabado, como una verdad absoluta. El educador planteará preguntas o cuestionamientos diferentes relacionados con lo que se pretende conocer a los cuales se les dará solución de forma conjunta, sin imposiciones. La enseñanza bioética debería construirse sobre la base de una problematización contextualizada.

\section{- Relaciones horizontales de poder}

En el diálogo problematizador, al establecer la condición de guía u organizador del proceso, el educador debe construir un tipo de relación con el educando en la que este no se sienta desposeído total de conocimientos y el educador con el poder total sobre dicho conocimiento. Para tanto es necesario establecer relaciones de poder horizontales con los educandos, que eliminen la polarización del poder del conocimiento en manos únicamente de los educadores. Al estar educador y educando en igualdad de condiciones ante las posibilidades de llegar al conocimiento verdadero el educando se sentirá con mejor disposición ante el diálogo.

\section{- La transdisciplinariedad}

En el proceso pedagógico de la educación en bioética debe preverse la necesidad y posibilidad del diálogo entre individuos formados en diversas y diferentes ramas del saber para proporcionar a educandos y educadores una integralidad en la búsqueda de la verdad. Integralidad que permitirá analizar la realidad desde la complejidad en que esta existe y se manifiesta.

\section{- La interculturalidad}

Partiendo del reconocimiento a las posibilidades epistémicas de todos los individuos debemos plantear que el proceso pedagógico en bioética no puede obviar el dialogo con personas de diferentes culturas; que no exonera la posibilidad de considerar incluso a aquellos que no tienen una formación académica reconocida pero si una experiencia práctica que le ha permitido conocer a profundidad sobre un tema determinado.

\section{- La concientización}

Por ultimo desde el punto de vista pedagógico y como colofón de todos los postulados anteriores está el que toda metodología para la educación en bioética debe tener como objetivo general máximo el logro de una formación transdisciplinar del individuo. Formación que le permita lograr la concientización de su yo interno interpretando correctamente su realidad y el papel que desempeña en esta. La concientización es necesaria para luchar contra todo lo que pueda impedir su desarrollo, porque al lograr su desarrollo, puede contribuir al desarrollo social. Quienes trabajamos por una educación bioética pertinente, deberíamos preguntarnos si es posible arribar a los resultados esperados sin que la educación bioética sea en sí misma un proceso de concientización, toma de conciencia del ser propio, que se desenvuelve en un contexto hoy global y local.

Es pertinente aquí preguntarnos ¿no es ese uno de los propósitos de la educación bioética? 0 ¿deberíamos reducirla al desarrollo de habilidades para analizar situaciones de conflicto y valorar posibles salidas instrumentales? El propósito de la problematización en la educación bioética no debe girar en torno a la didáctica de la enseñanza, sino en torno al objetivo, que consiste en que los individuos tomen conciencia de su situación. La situación en este caso implica el reconocimiento del lugar de la tecnología, las relaciones de dominación, la justicia etc. en nuestra vida contemporánea como sociedades que tienen por delante el reto de la supervivencia y el manejo adecuado del conocimiento para garantizarla.

\section{Premisas éticas}

Pudiera parecer vicioso exponer la necesidad de declarar postulados básicos en este sentido. Sin embargo entendemos necesario hacerlo, para hacer visibles algunas aristas frecuentemente obviadas de principios éticos fundamentales relacionados con los aspectos epistemológicos y pedagógicos expuestos con anterioridad. Principios éticos que trataremos cada uno como un sustento ético fundamental.

\section{- Humildad}

Este principio se refiere esencialmente a la necesidad de que los miembros del proceso de enseñanza en todos los pasos del mismo y desde todas las posiciones posibles mantengan una postura humilde. Postura que les permita aceptar que es posible que se hayan equivocado que no existe el control absoluto del conocimiento por parte de ningún individuo. Así mismo actúen en consecuencia con 
este principio dejándose educar en todo momento siempre que la intervención del resto de los interlocutores participantes del diálogo se lo permita.

Está claro que la educación en bioética necesita incorporar la interpretación e instrumentación pedagógica del concepto de humildad, tanto en las prácticas diferentes la instrucción escolarizada, como en la educación bioética en general. La Humildad es un principio básico en la triada de postulados éticos que analizaremos por que - como veremos a continuación - el cumplimiento de este sentará las bases para un comportamiento responsable y autónomo.

\section{- Responsabilidad}

La responsabilidad es un principio que se hace mucho más fuerte si se desprende de un comportamiento humilde. El reconocer que usted como educador o educando puede equivocarse le permite asumir con mucha más seriedad el acto educativo y por ende luchar con todos los mecanismos a su alcance para que sean fructíferas las consecuencias de sus actos. Dejándose incluso guiar por todo aquel que pueda contribuir a perfeccionar el proceso porque ha asumido la responsabilidad del resultado de sus acciones.

\section{- Autonomía}

Aun cuando en múltiples ocasiones, sobre todo desde la posición de educando, se olvida la posibilidad y necesidad de exigir la autonomía propia es mucho más frecuente el que tanto el educando como el educador defiendan su derecho a la autonomía en el proceso de elaboración del conocimiento. Sin embargo de lo que aquí se trata, en correspondencia con una actitud humilde, es que luchemos por esa autonomía propia a partir del respeto a la autonomía ajena. No es posible lograr llegar a la sabiduría de cómo manejar el conocimiento, a la que se refiere Potter si no se respeta y se considera como tal todo conocimiento, venga desde la posición y la cultura que venga.

Por otra parte, la comprensión freireana de la autonomía debería incorporarse al análisis de las diversas propuestas principialistas, que suelen desligar la autonomía del individuo de su responsabilidad colectiva como miembro de la comunidad humana.

\section{Consideraciones finales}

A partir de los argumentos expuestos estamos en condiciones de concluir que la bioética potteriana es una disciplina de nuevo tipo a partir de que exige, desde la doble condición contenida en el adjetivo de puente, el romper con el ideal clásico del conocimiento, para mediante la transdisciplinariedad lograr discernir una sabiduría en el manejo del conocimiento que nos permita construir con responsabilidad el camino hacia el futuro.

La concepción pedagógica freireana constituye una crítica al modelo pedagógico tradicional que desde su comprometimiento con los oprimidos tiene en esencia como característica fundamental su carácter democrático, basamento de su condición dialógica, reflexiva, humilde, sensible, interdisciplinar y política; defensora de dos principios éticos fundamentales: la responsabilidad y la autonomía y con un objetivo único: La concientización del educando como la única forma de lograr su condición de liberadora. La educación bancaria no es una forma pertinente para desplegar la educación bioética porque contraviene los ideales que le sirven de base y pone en riesgo que se alcancen los objetivos planteados por la misma.

El explicitar los elementos teóricos existentes en ambas teorías permitió establecer diálogo enriquecedor entre las mismas. Diálogo que va desde la existencias de conceptos validados por una de las teorías, posibles de aplicar en la otra; pasando por una lógica dialéctica común que se constituye en un pensamiento crítico sobre la forma en que tradicionalmente se manejó el conocimiento; hasta llegar a la existencia de un ideal común. Ideal que se caracteriza por su carácter democrático, su condición dialógica, sensible, interdisciplinar, humilde, ética y su alcance político. La educación freireana es una forma pertinente para el despliegue de la educación bioética porque habilita el diálogo de saberes y permite que se alcancen los objetivos propios de la educación bioética: habilita para alcanzar sabiduría en el manejo del conocimiento y ponerlo en función de la supervivencia humana.

\section{Referências}

1. Potter VR. Bioethics bridge to the future. New Jersey: Prentice-Hall; 1971.

2. Potter VR. Global bioethics: building on the Leopold legacy. East Lansing: Michigan State University Press; 1988.

3. Sariego JRA. Bioética para la sustentabilidad. La Habana: Publicaciones Acuario; 2002. 
4. Sariego JRA. Los árboles y el bosque: texto y contexto bioético cubano. La Habana: Publicaciones Acuario; 2009. p. 157.

5. Freire P. Conscientização e alfabetização: uma nova visão do processo. Estudos Universitários: revista de cultura da Universidade do Recife. 1963;4:5-22.

6. Freire P. Pedagogia do oprimido. $18^{\mathrm{a}}$ ed. Rio de Janeiro: Paz e Terra; 1988.

7. Freire P. Educação como prática da liberdade. Rio de Janeiro: Paz e Terra; 1967.

8. Freire P. Educação e mudança. São Paulo: Paz e Terra; 1979.

9. Freire P. Pedagogia da esperança. São Paulo: Paz e Terra; 1992.

10. Freire P. Pedagogia da autonomia. São Paulo: Paz e Terra; 2001.

11. Pérez E. Conferencia en el curso de educación dialógica. Tercera edición de la maestría en bioética. La Habana: CESBH/UH; 2011.

12. Delgado CJ. Diálogo de saberes para una reforma del pensamiento y la enseñanza en América Latina: Morin-Potter-Freire. Estudios (México): filosofía, historia, letras. 2010;8(93):30.

13. Delgado CJ. Op. cit. p. 23-45.

14. Delgado CJ. Op. cit. p. 37.
Recebido: 15.7 .2012

Revisado: 18.12 .2012

Aprovado: 15. 2.2013 